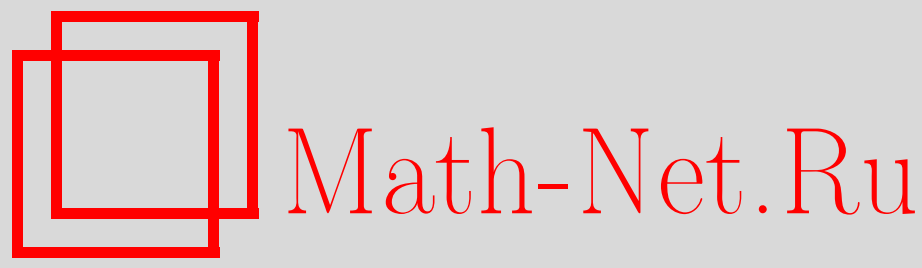

М. Э. Муминов, А. М. Хуррамов, О кратности виртуального уровня нижнего края непрерывного спектра одного двухчастичного гамильтониана на решетке, $Т M \Phi$, 2014, том 180, номер 3, 329-341

DOI: https://doi.org/10.4213/tmf8624

Использование Общероссийского математического портала Math-Net.Ru подразумевает, что вы прочитали и согласны с пользовательским соглашением http: //www . mathnet.ru/rus/agreement

Параметры загрузки:

IP : 34.239 .49 .27

26 апреля 2023 г., 10:57:48

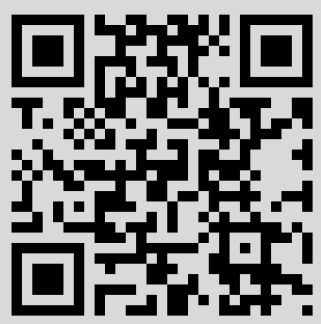


Том 180, № 3

сентябрь, 2014

(C) 2014 г. М. Э. Муминов*†, А. М. Хуррамов*

\title{
О КРАТНОСТИ ВИРТУАЛЬНОГО УРОВНЯ НИЖНЕГО КРАЯ НЕПРЕРЫВНОГО СПЕКТРА ОДНОГО ДВУХЧАСТИЧНОГО ГАМИЛЬТОНИАНА HA PEШETKE
}

\begin{abstract}
Рассматривается система двух произвольных квантовых частиц на трехмерной решетке со специальными дисперсионными функциями (описывающими перенос частицы с узла на узел), взаимодействующих с помощью выбранного потенциала притяжения. Изучена зависимость числа собственных значений семейства операторов $h(k)$ от энергии взаимодействия частиц и полного квазиимпульса $k \in \mathbb{T}^{3}$ (где $\mathbb{T}^{3}$ - трехмерный тор). В зависимости от энергии взаимодействия частиц найдены условия, при которых левый край непрерывного спектра является многократным и одновременно и виртуальным уровнем, и собственным значением оператора $h(\mathbf{0})$.
\end{abstract}

Ключевые слова: двухчастичный гамильтониан на решетке, виртуальный уровень, кратность виртуального уровня, собственное значение.

DOI: $10.4213 / \operatorname{tmf} 8624$

\section{1. ВВЕДЕНИЕ}

В непрерывном случае изучение спектральных свойств полного гамильтониана системы двух частиц сводится к изучению двухчастичного оператора Шредингера с помощью выделения энергии движения центра масс, при этом одночастичные связанные состояния суть собственные векторы оператора энергии с отделенным полным импульсом [1] (такой оператор фактически не зависит от значений полного импульса). На решетке выделению центра масс системы отвечает реализация гамильтониана как "расслоенного" оператора, т. е. прямого интеграла семейства операторов $h(k)$ энергии двух частиц, зависящих от значений полного квазиимпульса $k \in \mathbb{T}^{d}$ ( $\mathbb{T}^{d}-d$-мерный тор) [2], [3]. Конечность и бесконечность числа дискретных собственных значений многочастичных систем зависят от числа виртуальных

${ }^{*}$ Самаркандский государственный университет, Самарканд, Узбекистан.

E-mail: mmuminov@mail.ru,xurramov@mail.ru

${ }^{\dagger}$ Universiti Technologi Malaysia, Johor, Malaysia 
уровней подсистем. В трехчастичной системе, если две или три двухчастичные подсистемы имеют виртуальные уровни, дискретный спектр гамильтониана бесконечен [4], [5], и если в системе таких подсистем не более одной, то дискретный спектр гамильтониана конечен [6], [7].

Вопрос о существовании виртуального уровня для системы $n$ частиц $(n>3)$ рассматривался в работах ЖКислина [7], [8]. Для системы двух частиц на решетке существование виртуального уровня изучалось в работах [3], [9], [10] в случае, когда дисперсионные соотношения частиц линейно зависимы и имеют единственный невырожденный минимум в нуле.

Двухчастичные гамильтонианы на решетке исследованы в работах [11], [12]. В работе [11] показано появление уровней связанных состояний, отстоящих от непрерывного спектра на определенное расстояние, при некоторых значениях полного квазиимпульса системы. Спектральные свойства двухчастичного оператора в зависимости от полного квазиимпульса изучены в работе [12].

В работе [3] доказано, что в случае, когда оператор $h(\mathbf{0})$ имеет виртуальный уровень на левом крае существенного спектра, дискретный спектр оператора $h(k)$, лежащий левее существенного спектра, является не пустым при всех $k \in \mathbb{T}^{d} \backslash\{\boldsymbol{0}\}$. В работе [13] в предположении, что дисперсионные соотношения частиц $\varepsilon_{1}(\cdot)$ и $\varepsilon_{2}(\cdot)-$ линейно зависимые функции, доказано, что из положительности $h(\mathbf{0})$ следует положительность $h(k)$ при всех $k \in \mathbb{T}^{3} \backslash\{\mathbf{0}\}$ (где $\mathbb{T}^{3}$ - трехмерный тор).

В работе [14] рассмотрена система двух частиц на трехмерной решетке с некоторой дисперсионной функцией, описывающей перенос частицы с узла на соседний узел. Частицы взаимодействуют с помощью потенциала притяжения только в ближайших соседних узлах. Изучены спектральные свойства семейства операторов $h(k)$ в зависимости от энергии взаимодействия частиц и полного квазиимпульса $k \in \mathbb{T}^{3}$. Кроме того, в зависимости от энергии взаимодействия частиц найдены условия, при которых оператор $h(\mathbf{0})$ имеет простой, двукратный и трехкратный виртульные уровни в нуле.

Настоящая работа является обобщением работы [14]. Мы изучаем двухчастичный оператор Шредингера $h(k), k \in \mathbb{T}^{3}$, соответствующий системе двух частиц на трехмерной решетке, где в качестве потенциала берется некоторый $6 N$-мерный интегральный оператор и в зависимости от $N$ выбрана дисперсионная функция. Изучается вопрос о существовании или отсутствии собственных значений семейства операторов $h(k)$ в зависимости от энергии взаимодействия частиц и полного квазиимпульса $k$. Кроме того, в зависимости от энергии взаимодействия частиц найдены условия, при которых оператор $h(\mathbf{0})$ имеет на левом крае существенного спектра одновременно и $3 N$-кратный виртульный уровень, и $3 N$-кратное собственное значение (см. ниже замечания 1,2 ). Следует отметить, что рассматриваемый оператор при $N=1$ совпадает с оператором из работы [14].

Для существования бесконечного числа связанных состояний (эффекта Ефимова) гамильтониана системы трех частиц, взаимодействующих на трехмерной решетке с помощью парных контактных потенциалов притяжения, важно достижение виртуального уровня в нуле двухчастичных подгамильтонианов $h_{\alpha}(\mathbf{0}), \alpha=1,2,3$, а также положительность $h_{\alpha}(k), k \in \mathbb{T}^{3} \backslash\{\mathbf{0}\}, \alpha=1,2,3$. В работах [9], [10], [15], посвященных эффекту Ефимова, виртуальные уровни двухчастичных подгамильтонианов $h_{\alpha}(\mathbf{0})$, 
$\alpha=1,2,3$, являются простыми (однократными, см. ниже определение 1). Поэтому с учетом результатов настоящей работы появляется новая задача о существовании или отсутствии эффекта Ефимова для трехчастичного дискретного оператора Шредингера в случае, когда двухчастичные подгамильтонианы достигают кратного виртуального уровня в нуле.

\section{2. ФОРМУЛИРОВКА ОСНОВНЫХ РЕЗУЛЬТАТОВ}

Пусть $\mathbb{Z}^{3}$ - трехмерная целочисленная решетка, $\left(\mathbb{Z}^{3}\right)^{2}=\mathbb{Z}^{3} \times \mathbb{Z}^{3}$ - декартова степень $\mathbb{Z}^{3}, l_{2}\left(\left(\mathbb{Z}^{3}\right)^{2}\right)$ - гильбертово пространство квадратично-суммируемых функций, определенных на $\left(\mathbb{Z}^{3}\right)^{2}$. Рассмотрим координатное представление гамильтониана системы двух произвольных частиц, взаимодействующих с парным короткодействующим потенциалом $\hat{v}(\cdot)$ на трехмерной решетке, действующего в пространстве $l_{2}\left(\left(\mathbb{Z}^{3}\right)^{2}\right)$ :

$$
\hat{h}=\hat{h}_{0}-\hat{v}
$$

где $\hat{h}_{0}$ и $\hat{v}$ действуют по правилам

$$
\begin{aligned}
\left(\hat{h}_{0} \hat{\psi}\right)\left(n_{1}, n_{2}\right) & =\sum_{s \in \mathbb{Z}^{3}}\left[\hat{\varepsilon}_{1}(s) \hat{\psi}\left(n_{1}+s, n_{2}\right)+\hat{\varepsilon}_{2}(s) \hat{\psi}\left(n_{1}, n_{2}+s\right)\right], \\
(\hat{v} \hat{\psi})\left(n_{1}, n_{2}\right) & =\hat{v}\left(n_{1}-n_{2}\right) \hat{\psi}\left(n_{1}, n_{2}\right) .
\end{aligned}
$$

Здесь $\hat{\varepsilon}_{1}(s)$ и $\hat{\varepsilon}_{2}(s)$ - вещественнозначные четные функции, описывающие перенос частицы с узла на соседний узел,

$$
\hat{\varepsilon}_{i}(s)= \begin{cases}\frac{3}{m_{i}} & \text { при } \quad s=0, \\ -\frac{1}{2 m_{i}} & \text { при } \quad s= \pm 2 n e_{\alpha}, \quad \alpha=1,2,3, \\ 0 & \text { в остальных случаях, }\end{cases}
$$

и функция $\hat{v}(\cdot)$ задается следующей формулой:

$$
\hat{v}(s)= \begin{cases}4 \pi^{3} \mu_{l \alpha} & \text { при } \quad s= \pm l e_{\alpha}, \quad l=\overline{1, N}, \quad \alpha=1,2,3, \\ 0 & \text { в остальных случаях }\end{cases}
$$

где $m_{i}>0$ - масса $i$-й частицы, $i=1,2, \mu_{l \alpha}>0, e_{\alpha}, \alpha=1,2,3,-$ единичные орты, $n$ - натуральное число.

Отметим, что рассматриваемый оператор $\hat{h}$ является ограниченным и самосопряженным в $l_{2}\left(\left(\mathbb{Z}^{3}\right)^{2}\right)$.

Пусть $T=(-\pi, \pi], L_{2}\left(\mathbb{T}^{3}\right)$ - гильбертово пространство всех квадратично-интегрируемых функций, определенных на $\mathbb{T}^{3}$. C помощью преобразования Фурье [3], [13]

$$
\Im: l_{2}\left(\left(\mathbb{Z}^{3}\right)^{2}\right) \rightarrow L_{2}\left(\left(\mathbb{T}^{3}\right)^{2}\right), \quad(\Im \hat{f})(p)=\frac{1}{8 \pi^{3}} \sum_{s \in\left(\mathbb{Z}^{3}\right)^{2}} \hat{f}(s) e^{-i p s}
$$

получим импульсное представление $h$ оператора $\hat{h}$, т. е. $h=\Im \hat{h} \Im^{-1}$. Далее разложим оператор $h$ в прямой операторный интеграл

$$
h=\int_{\mathbb{T}^{3}} \oplus h(k) d k,
$$


где $h(k), k \in \mathbb{T}^{3},-$ самосопряженный оператор, действующий в $L_{2}\left(\mathbb{T}^{3}\right)$ по формуле

$$
h(k)=h_{0}(k)-\mathbf{v},
$$

здесь $h_{0}(k)$ - оператор умножения на функцию

$$
\mathcal{E}_{k}(p)=\frac{1}{m_{1}} \varepsilon(p)+\frac{1}{m_{2}} \varepsilon(p-k), \quad \varepsilon(p)=\sum_{s \in \mathbb{Z}^{3}} \hat{\varepsilon}(s) e^{i p s}=\sum_{i=1}^{3}\left(1-\cos 2 n p_{i}\right),
$$

и $\mathbf{v}$ - интегральный оператор с ядром

$$
v(p-q)=\frac{1}{(2 \pi)^{3}} \sum_{s \in \mathbb{Z}^{3}} \hat{v}(s) e^{-i(p-q) s}=\sum_{l=1}^{N} \sum_{i=1}^{3} \mu_{l i} \cos l\left(p_{i}-q_{i}\right) .
$$

Отметим, что из теоремы Вейля о существенном спектре [16] следует, что существенный спектр $\sigma_{\text {ess }}(h(k))$ оператора $h(k)$ не меняется при компактном возмущении $\mathbf{v}$ и совпадает со спектром невозмущенного оператора $h_{0}(k)$. При этом $\sigma_{\text {ess }}(h(k))$ совпадает с областью значений функции $\mathcal{E}_{k}(\cdot)$, т. е.

$$
\sigma_{\mathrm{ess}}(h(k))=\sigma\left(h_{0}(k)\right)=[m(k), M(k)],
$$

где $m(k)=\min _{p} \mathcal{E}_{k}(p), M(k)=\max _{p} \mathcal{E}_{k}(p)$.

Поскольку $\mathbf{v} \geqslant 0$, то

$$
\sup (h(k) f, f) \leqslant \sup \left(h_{0}(k) f, f\right)=M(k)(f, f), \quad f \in L_{2}\left(\mathbb{T}^{3}\right) .
$$

Поэтому оператор $h(k)$ не имеет собственного значения, лежащего правее существенного спектра, т. е.

$$
\sigma(h(k)) \cap(M(k), \infty)=\varnothing .
$$

В дальнейшем будем считать, что

$$
n=\left\{\begin{array}{lll}
\operatorname{HOK}\{2,4, \ldots, 2(N-1)\} & \text { при } & N>1 \\
1 & \text { при } & N=1
\end{array}\right.
$$

где НОК - наименьшее общее кратное. Следует отметить, что если $N$ представляется в виде степени простого числа, то число $n / 2 N$ является дробным. В противном случае число $n / 2 N$ является натуральным.

ПРЕДПОЛОЖЕНИЕ 1. Предположим, что $m=m_{1}=m_{2} u k \in \Pi$, где П есть множество тех $k=\left(k_{1}, k_{2}, k_{3}\right) \in \mathbb{T}^{3}$, для которых $k_{\alpha}=-\pi / 2 n$ или $k_{\alpha}=\pi / 2 n$ хотя би для одного $\alpha \in\{1,2,3\}$.

При условии невыполнения предположения 1 положим (см. ниже лемму 3)

$$
\mu^{0}(k)=\frac{1}{d(k ; m(k))}, \quad \mu_{N i}^{c}(k)=\frac{1}{c_{N i}(k ; m(k))}, \quad \mu_{N i}^{s}(k)=\frac{1}{s_{N i}(k ; m(k))},
$$


где

$$
\begin{aligned}
d(k ; z)= & \frac{1}{2} \int_{\mathbb{T}^{3}} \frac{d s}{\widetilde{\mathcal{E}}_{k}(s)-z}, \quad c_{N i}(k ; z)=\int_{\mathbb{T}^{3}} \frac{\cos ^{2} N s_{i} d s}{\widetilde{\mathcal{E}}_{k}(s)-z}, \quad s_{N i}(k ; z)=\int_{\mathbb{T}^{3}} \frac{\sin ^{2} N s_{i} d s}{\widetilde{\mathcal{E}}_{k}(s)-z} \\
& \widetilde{\mathcal{E}}_{k}(p)=\sum_{i=1}^{3}\left(\frac{1}{m_{1}}+\frac{1}{m_{2}}-\sqrt{\frac{1}{m_{1}^{2}}+\frac{2}{m_{1} m_{2}} \cos 2 n k_{i}+\frac{1}{m_{2}^{2}}} \cos 2 n p_{i}\right) .
\end{aligned}
$$

Отметим, что если $n / 2 N$ - натуральное (дробное) число, то $c_{N i}(k ; z)=s_{N i}(k ; z)$ $\left(c_{N i}(k ; z)>s_{N i}(k ; z)\right)[14]$. Поэтому $\mu_{N i}^{c}(k)<\mu_{N i}^{s}(k)$ при дробном $n / 2 N$.

ТЕорема 1. Пусть не выполняется предположение $1, u n / 2 N$ - натуральное (дробное) число. Тогда для любого $\mu \in \mathbb{R}_{+}^{3 N}, \mu=\left(\mu^{(1)}, \ldots, \mu^{(N)}\right), \mu^{(l)}=\left(\mu_{l 1}, \mu_{l 2}, \mu_{l 3}\right)$, оператор $h(k)$ имеет с учетом кратности ровно

$$
2 \sum_{l=1}^{N} \sum_{i=1}^{3} \alpha(l i) \quad\left(\text { соответственно } 2 \sum_{l=1}^{N-1} \sum_{i=1}^{3} \alpha(l i)+\sum_{i=1}^{3} \beta(N i)\right)
$$

собственных значений, лежащих левее существенного спектра, где

$$
\begin{gathered}
\alpha(l i)=\left\{\begin{array}{lll}
0 & n p u & \mu_{l i} \in\left(0, \mu^{0}(k)\right], \\
1 & n p u & \mu_{l i} \in\left(\mu^{0}(k), \infty\right),
\end{array}\right. \\
\beta(N i)=\left\{\begin{array}{lll}
0 & n p u & \mu_{N i} \in\left(0, \mu_{N i}^{c}(k)\right], \\
1 & n p u & \mu_{N i} \in\left(\mu_{N i}^{c}(k), \mu_{N i}^{s}(k)\right], \\
2 & n p u & \mu_{N i} \in\left(\mu_{N i}^{s}(k), \infty\right) .
\end{array}\right.
\end{gathered}
$$

Теорема 2. Пусть выполняется предположение 1, и $n / 2 N$ - натуралъное число. Тогда для любого $\mu \in \mathbb{R}_{+}^{3 N}, \mu=\left(\mu^{(1)}, \ldots, \mu^{(N)}\right), \mu^{(l)}=\left(\mu_{l 1}, \mu_{l 2}, \mu_{l 3}\right)$, оператор $h(k)$ имеет с учетом кратности ровно $6 N$ собственных значений, лежащих левее существенного спектра.

Пусть $C\left(\mathbb{T}^{3}\right)$ - банахово пространство непрерывных (периодических) функций на $\mathbb{T}^{3}$, и $G$ - интегральный оператор (Бирмана-Швингера) с ядром

$$
G(p, q)=v(p-q)\left(\mathcal{E}_{\mathbf{0}}(q)\right)^{-1}, \quad p, q \in \mathbb{T}^{3} .
$$

ОПРЕДЕЛЕНИЕ 1. Если единица является собственным значением оператора $G$ и соответствующая собственная функция $\psi$ удовлетворяет условию

$$
\frac{\psi(p)}{\mathcal{E}_{\mathbf{0}}(p)} \in L_{1}\left(\mathbb{T}^{3}\right) \backslash L_{2}\left(\mathbb{T}^{3}\right),
$$

то говорят, что оператор $h(\mathbf{0})$ имеет виртуальный уровень в нуле (на левом крае существенного спектра). Число таких линейно независимых собственных векторов $\psi$ оператора $G$ назовем кратностъю виртуального уровня оператора $h(\mathbf{0})$.

Аналогично лемме 1 работы [14] доказывается 
Лемма 1. Пусть $n / 2 N$ - натуралъное (дробное) число, $\mu_{l i} \in\left[0, \mu^{0}(\mathbf{0})\right]$ (здесъ $\left.\mu_{l i} \in\left[0, \mu^{0}(\mathbf{0})\right], \mu_{N i} \in\left[0, \mu_{N}^{0}(\mathbf{0})\right]\right)$ для каждого $l \in\{1, \ldots, N\}(l \in\{1, \ldots, N-1\})$ $u i \in\{1,2,3\}$. Тогда оператор $h(\mathbf{0})$ не имеет собственного значения, лежащего левее существенного спектра.

Из леммы 1 следует, что $h(\mathbf{0}) \geqslant 0$. Поэтому в этом случае из теоремы 1 работы [13] вытекает, что $h(k) \geqslant 0$ для всех $k \in \mathbb{T}^{3}$, т. е. оператор $h(k)$ не имеет отрицательного собственного значения при $k \in \mathbb{T}^{3}$.

Теорема 3. Пусть $n / 2 N$ - натуральное (дробное) число. Тогда для любого значения $\mu \in \mathbb{R}_{+}^{3 N}, \mu=\left(\mu^{(1)}, \ldots, \mu^{(N)}\right), \mu^{(l)}=\left(\mu_{l 1}, \mu_{l 2}, \mu_{l 3}\right)$, нуль является одновременно и кратным собственным значением с кратностью

$$
\sum_{l=1}^{N} \sum_{i=1}^{3} \alpha(l i) \quad\left(\text { соответственно } \sum_{l=1}^{N-1} \sum_{i=1}^{3} \alpha(l i)\right)
$$

и кратным виртуалъным уровнем оператора $h(\mathbf{0})$ и кратностъю

$$
\sum_{l=1}^{N} \sum_{i=1}^{3} \alpha(l i) \quad\left(\text { соответственно } \sum_{l=1}^{N-1} \sum_{i=1}^{3} \alpha(l i)+\sum_{i=1}^{3} \beta(N i)\right)
$$

где

$$
\alpha(l i)=\left\{\begin{array}{lll}
0 & n p u & \mu_{l i} \in\left[0 ; \mu^{0}(\mathbf{0})\right), \\
1 & n p u & \mu_{l i} \in\left\{\mu^{0}(\mathbf{0})\right\},
\end{array} \quad \beta(N i)=\left\{\begin{array}{lll}
0 & n p u & \mu_{N i} \in\left[0 ; \mu_{N 1}^{0}(\mathbf{0})\right) \\
1 & n p u & \mu_{N i} \in\left\{\mu_{N 1}^{0}(\mathbf{0})\right\}
\end{array}\right.\right.
$$

ЗАмЕчАниЕ 1 . В условиях теоремы 3 в случае

$$
\sum_{l=1}^{N} \sum_{i=1}^{3} \alpha(l i)=0 \quad\left(\text { соответственно } \sum_{l=1}^{N-1} \sum_{i=1}^{3} \alpha(l i)+\sum_{i=1}^{3} \beta(N i)=0\right)
$$

считается, что $z=0$ не является ни виртуальным уровнем, ни собственным значением оператора $h(\mathbf{0})$. Если число $\mu_{l i}=\mu^{0}(\mathbf{0})\left(\mu_{l i}=\mu^{0}(\mathbf{0}), \mu_{N i}=\mu_{N 1}^{0}(\mathbf{0})\right)$ для всех $l \in\{1, \ldots, N\}(l \in\{1, \ldots, N-1\})$ и $i \in\{1,2,3\}$, то $z=0$ является одновременно и $3 N$-кратным виртуальным уровнем, и $3 N$-кратным собственным значением $(3 N$-кратным виртуальным уровнем и $3(N-1)$-кратным собственным значением) оператора $h(\mathbf{0})$. В этом случае единица является $6 N$-кратным собственным значением оператора $G$, а соответствующие собственные функции имеют вид $\varphi_{l i}=\cos l p_{i}$, $\psi_{l i}=\sin l p_{i}, l=1, \ldots, N, i=1,2,3$.

ЗАмечАниЕ 2. Следует отметить, что рассматриваемый оператор при $N=1$ совпадает с оператором из работы [14]. В этом случае $n / 2 N=1 / 2$. 


\section{3. СОБСТВЕННЫЕ ЗНАЧЕНИЯ $H(K)$}

В этом разделе докажем теоремы 1 и 2.

Введем оператор $\tilde{h}(k)$, действующий в $L_{2}\left(\mathbb{T}^{3}\right)$ по формуле

$$
\tilde{h}(k)=\tilde{h}_{0}(k)-\mathbf{v}
$$

где $\tilde{h}_{0}(k)$ - оператор умножения на функцию

$$
\widetilde{\mathcal{E}}_{k}(p)=\sum_{i=1}^{3}\left(\frac{1}{m_{1}}+\frac{1}{m_{2}}-\sqrt{\frac{1}{m_{1}^{2}}+\frac{2}{m_{1} m_{2}} \cos 2 n k_{i}+\frac{1}{m_{2}^{2}}} \cos 2 n p_{i}\right) .
$$

Определим унитарный оператор $U: L_{2}\left(\mathbb{T}^{3}\right) \rightarrow L_{2}\left(\mathbb{T}^{3}\right)$ :

$$
(U f)(p)=f\left(p-\frac{1}{2 n} \theta(k)\right)
$$

где

$$
\begin{aligned}
\theta(k) & =\left(\theta_{1}\left(k_{1}\right), \theta_{2}\left(k_{2}\right), \theta_{3}\left(k_{3}\right)\right), \\
\theta_{i}\left(k_{i}\right) & =\arccos \frac{1 / m_{1}+\left(1 / m_{2}\right) \cos 2 n k_{i}}{\sqrt{1 / m_{1}^{2}+\left(2 / m_{1} m_{2}\right) \cos 2 n k_{i}+1 / m_{2}^{2}}}, \quad i=1,2,3 .
\end{aligned}
$$

Тогда

$$
\left(U^{-1} f\right)(p)=f\left(p+\frac{1}{2 n} \theta(k)\right), \quad f \in L_{2}\left(\mathbb{T}^{3}\right) .
$$

Лемма 2. Оператор $h(k)$ является унитарно-эквивалентным оператору $\tilde{h}(k)$, m.e.

$$
\tilde{h}(k)=U^{-1} h(k) U=\tilde{h}_{0}(k)-\mathbf{v} .
$$

ДокАЗАТЕЛЬСТво. Поскольку имеет место представление

$$
\mathcal{E}_{k}(p)=\sum_{i=1}^{3}\left[\frac{1}{m_{1}}+\frac{1}{m_{2}}-\sqrt{\frac{1}{m_{1}^{2}}+\frac{2}{m_{1} m_{2}} \cos 2 n k_{i}+\frac{1}{m_{2}^{2}}} \cos \left(2 n p_{i}-\theta_{i}\left(k_{i}\right)\right)\right],
$$

то

$$
\begin{aligned}
\left(h_{0}(k) U f\right)(p)=\sum_{i=1}^{3} & \left(\frac{1}{m_{1}}+\frac{1}{m_{2}}-\sqrt{\frac{1}{m_{1}^{2}}+\frac{2}{m_{1} m_{2}} \cos 2 n k_{i}+\frac{1}{m_{2}^{2}}} \cos \left(2 n p_{i}-\theta_{i}\left(k_{i}\right)\right)\right) \times \\
& \times f\left(p-\frac{1}{2 n} \theta(k)\right) .
\end{aligned}
$$


Отсюда легко получим

$$
\left(U^{-1} h_{0}(k) U f\right)(p)=\sum_{i=1}^{3}\left(\frac{1}{m_{1}}+\frac{1}{m_{2}}-\sqrt{\frac{1}{m_{1}^{2}}+\frac{2}{m_{1} m_{2}} \cos 2 n k_{i}+\frac{1}{m_{2}^{2}}} \cos 2 n p_{i}\right) f(p),
$$

T. e.

$$
U^{-1} h_{0}(k) U=\tilde{h}_{0}(k) .
$$

Ясно, что

$$
\begin{aligned}
\left(U^{-1} \mathbf{v} U f\right)(p) & =U^{-1}\left(\int_{\mathbb{T}^{3}} v(s-p) f\left(s-\frac{1}{2 n} \theta(k)\right) d s\right)= \\
& =\int_{\mathbb{T}^{3}} v\left(s-\left(p+\frac{1}{2 n} \theta(k)\right)\right) f\left(s-\frac{1}{2 n} \theta(k)\right) d s .
\end{aligned}
$$

В последнем интеграле, произведя замену $s-\theta(k) / 2 n=t$, имеем

$$
\left(U^{-1} \mathbf{v} U f\right)(p)=\int_{\mathbb{T}^{3}} v(t-p) f(t) d t,
$$

т. e.

$$
U^{-1} \mathbf{v} U=\mathbf{v}
$$

Лемма доказана.

ЛЕмма 3. Пусть не выполняется предположение 1. Тогда интеграл

$$
\int_{\mathbb{T}^{3}} \frac{\varphi(s) d s}{\widetilde{\mathcal{E}}_{k}(s)-m(k)}
$$

сходится для всех $\varphi \in C\left(\mathbb{T}^{3}\right)$.

ДокАЗАТЕЛЬСтво. Поскольку не выполняется предположение 1 , то либо $m_{1} \neq m_{2}$ и $k \in \mathbb{T}^{3}$, либо $m=m_{1}=m_{2}$ и $k \notin \Pi$. В обоих случаях

$$
\frac{1}{m_{1}^{2}}+\frac{2}{m_{1} m_{2}} \cos 2 n k_{i}+\frac{1}{m_{2}^{2}}=\left(\frac{1}{m_{1}}-\frac{1}{m_{2}}\right)^{2}+\frac{2}{m_{1} m_{2}}\left(1+\cos 2 n k_{i}\right) \neq 0 .
$$

Поэтому $m(k)$ является невырожденной точкой минимума функции $\widetilde{\mathcal{E}}_{k}(\cdot)$ и

$$
\left\{p: \widetilde{\mathcal{E}}_{k}(p)=m(k)\right\}=\left\{p=\left(p_{1}^{(l)}, p_{2}^{(m)}, p_{3}^{(s)}\right), p_{i}^{(s)}=\frac{s \pi}{n}, s=0, \pm 1, \pm 2, \ldots\right\} .
$$

Так как минимум функции $\widetilde{\mathcal{E}}_{k}(\cdot)$ невырожденный, то интеграл $(3)$ существует для всех $\varphi \in C\left(\mathbb{T}^{3}\right)$.

Лемма 4. Число $z, z<m(k)$, является собственным значением оператора $\tilde{h}(k)$ тогда и только тогда, когда $\Delta(k ; z)=0$, где

$$
\Delta(k ; z)= \begin{cases}\prod_{l=1}^{N} \prod_{\alpha=1}^{3}\left[1-\mu_{l \alpha} d(k ; z)\right]^{2} \quad \text { при натуральном } & \frac{n}{2 N}, \\ \prod_{l=1}^{N-1} \prod_{\alpha=1}^{3}\left[1-\mu_{l \alpha} d(k ; z)\right]^{2}\left[1-\mu_{N \alpha} c_{N \alpha}(k ; z)\right] \times \\ \quad \times\left[1-\mu_{N \alpha} s_{N \alpha}(k ; z)\right] \quad \text { nри дробном } \frac{n}{2 N} .\end{cases}
$$


При этом кратность нуля функции $\Delta(k ; \cdot)$ совпадает с кратностью собственного значения оператора $h(k)$.

ДокАЗАтЕльство. Пусть $z<m(k)$ - собственное значение оператора $\tilde{h}(k)$, и $f-$ соответствующий собственный вектор, т. е. уравнение

$$
\tilde{h}(k) f=z f
$$

имеет нетривиальное решение $f$. Отсюда получаем

$$
f=r_{0}(z) \mathbf{v} f
$$

где $r_{0}(z)$ - оператор умножения на функцию $1 /\left(\widetilde{\mathcal{E}}_{k}(p)-z\right)$. Введем обозначения

$$
\begin{aligned}
& \varphi_{l \alpha}=\int_{\mathbb{T}^{3}} \cos l s_{\alpha} f(s) d s, \\
& \psi_{l \alpha}=\int_{\mathbb{T}^{3}} \sin l s_{\alpha} f(s) d s,
\end{aligned}
$$

Тогда равенство (4) принимает вид

$$
f(p)=\frac{1}{\widetilde{\mathcal{E}}_{k}(p)-z} \sum_{l=1}^{N} \sum_{\alpha=1}^{3} \mu_{l \alpha}\left[\cos l p_{\alpha} \varphi_{l \alpha}+\sin l p_{\alpha} \psi_{l \alpha}\right]
$$

Подставляя выражение (6) в формулы (5) и пользуясь четностью функции $\widetilde{\mathcal{E}}_{k}(\cdot)$, получим систему уравнений

$$
\begin{aligned}
\varphi_{l \alpha} & =\int_{\mathbb{T}^{3}} \sum_{r=1}^{N} \sum_{\beta=1}^{3} \mu_{r \beta} \frac{\cos l s_{\alpha} \cos r s_{\beta} d s}{\widetilde{\mathcal{E}}_{k}(s)-z} \varphi_{r \beta}, \\
\psi_{l \alpha} & =\int_{\mathbb{T}^{3}} \sum_{r=1}^{N} \mu_{l \alpha} \frac{\sin l s_{\alpha} \sin r s_{\alpha} d s}{\widetilde{\mathcal{E}}_{k}(s)-z} \psi_{r \alpha},
\end{aligned}
$$

Отметим, что по определению числа $n$ число $n / 2 l$ является натуральным для всех $l=1, \ldots, N-1$. Отсюда следует, что функция $\widetilde{\mathcal{E}}_{k}(\cdot)$ является периодической по каждому аргументу, с периодом $\pi / 2 l$ для всех $l=1, \ldots, N-1$. Покажем, что

$$
\int_{\mathbb{T}^{3}} \frac{\cos l s_{\alpha} d s}{\widetilde{\mathcal{E}}_{k}(s)-z}=0, \quad l=1, \ldots, 2 N-1 .
$$

Если $l$ - нечетное (четное) число, то заменяя переменные $s_{\alpha}=t_{\alpha}+\pi\left(s_{\alpha}=t_{\alpha}+\pi / l\right)$, $s_{\beta}=t_{\beta}, s_{\gamma}=t_{\gamma}$, в интеграле в левой части равенства (8) имеем

$$
I_{l}(z)=-\int_{\mathbb{T}^{3}} \frac{\cos l t_{\alpha} d t}{\widetilde{\mathcal{E}}_{k}(t)-z}=-I_{l}(z) .
$$

Отсюда следует равенство (8). Из равенства (8) получим

$$
\int_{\mathbb{T}^{3}} \frac{\cos l s_{\alpha} \cos r s_{\beta} d s}{\widetilde{\mathcal{E}}_{k}(s)-z}=0, \quad \alpha \neq \beta, \quad l, r=1, \ldots, N .
$$


Из элементарных равенств

$$
\begin{aligned}
\cos l s_{\alpha} \cos r s_{\alpha} & =\frac{1}{2}\left[\cos (l+r) s_{\alpha}+\cos (l-r) s_{\alpha}\right], \\
\sin l s_{\alpha} \sin r s_{\alpha} & =\frac{1}{2}\left[\cos (l-r) s_{\alpha}-\cos (l+r) s_{\alpha}\right]
\end{aligned}
$$

и из равенства (8) получим

$$
\begin{aligned}
& \int_{\mathbb{T}^{3}} \frac{\cos l s_{\alpha} \cos r s_{\alpha}}{\widetilde{\mathcal{E}}_{k}(s)-z} d s=0, \\
& \int_{\mathbb{T}^{3}} \frac{\sin l s_{\alpha} \sin r s_{\alpha}}{\widetilde{\mathcal{E}}_{k}(s)-z} d s=0,
\end{aligned}
$$

В силу (9), (10) равенства (7) приобретают вид

$$
\begin{aligned}
& \varphi_{l \alpha}=\int_{\mathbb{T}^{3}} \mu_{l \alpha} \frac{\cos ^{2} l s_{\alpha} d s}{\widetilde{\mathcal{E}}_{k}(s)-z} \varphi_{l \alpha}, \\
& \psi_{l \alpha}=\int_{\mathbb{T}^{3}} \mu_{l \alpha} \frac{\sin ^{2} l s_{\alpha} d s}{\widetilde{\mathcal{E}}_{k}(s)-z} \psi_{l \alpha}, \quad l=1, \ldots, N, \quad \alpha=1,2,3 .
\end{aligned}
$$

При этом если $z<m(k)$ - собственное значение оператора $\tilde{h}(k)$, то

$$
\Delta(k ; z)=\prod_{l=1}^{N} \prod_{\alpha=1}^{3}\left[1-\mu_{l \alpha} \int_{\mathbb{T}^{3}} \frac{\cos ^{2} l s_{\alpha} d s}{\widetilde{\mathcal{E}}_{k}(s)-z}\right]\left[1-\mu_{l \alpha} \int_{\mathbb{T}^{3}} \frac{\sin ^{2} l s_{\alpha} d s}{\widetilde{\mathcal{E}}_{k}(s)-z}\right]=0 .
$$

Легко видеть, что согласно (9) для всякого $l \leqslant N(l \leqslant N-1)$ и при натуральном $n / 2 N$ (при дробном $n / 2 N$ ) имеет место равенство

$$
\int_{\mathbb{T}^{3}} \frac{\cos ^{2} l s_{\alpha} d s}{\widetilde{\mathcal{E}}_{k}(s)-z}=\int_{\mathbb{T}^{3}} \frac{\sin ^{2} l s_{\alpha} d s}{\widetilde{\mathcal{E}}_{k}(s)-z}=\frac{1}{2} \int_{\mathbb{T}^{3}} \frac{d s}{\widetilde{\mathcal{E}}_{k}(s)-z}=d(k ; z), \quad \alpha=1,2,3 .
$$

Обратно, пусть

$$
1-\mu_{l \alpha} \int_{\mathbb{T}^{3}} \frac{\cos ^{2} l s_{\alpha} d s}{\widetilde{\mathcal{E}}_{k}(s)-z}=0 \quad\left(1-\mu_{l \alpha} \int_{\mathbb{T}^{3}} \frac{\sin ^{2} l s_{\alpha} d s}{\widetilde{\mathcal{E}}_{k}(s)-z}=0\right)
$$

для некоторого $l$ и $z<m(k)$. Тогда функция $\cos l p_{\alpha} /\left(\widetilde{\mathcal{E}}_{k}(p)-z\right)\left(\sin l p_{\alpha} /\left(\widetilde{\mathcal{E}}_{k}(p)-z\right)\right)$ является собственной функцией оператора $\tilde{h}(k)$, соответствующей собственному значению $z<m(k)$. При этом кратность нуля функции $\Delta(k ; \cdot)$ совпадает с кратностью собственного значения оператора $\tilde{h}(k)$. Лемма доказана.

ДоКАЗАТЕЛЬСТво ТЕОРЕМЫ 1. Пусть не выполняется предположение 1. Тогда для всякого $k \in \mathbb{T}^{3}$ числа $\mu^{0}(k), \mu_{N i}^{c}(k), \mu_{N i}^{s}(k), i=1,2,3$, определенные по формулам (1), являются конечными. 
Имеют место соотношения

$$
\begin{aligned}
1-\mu_{l \alpha} d(k ; z)=\left\{\begin{array}{lll}
\geqslant 0 & \text { при } & \mu_{l \alpha} \in\left(0, \mu^{0}(k)\right] \quad \text { для всякого } z \in(-\infty, m(k)], \\
<0 & \text { при } & \mu_{l \alpha}>\mu^{0}(k), \quad z=m(k), \\
1 & \text { при } & z \rightarrow-\infty,
\end{array}\right. \\
1-\mu_{N \alpha} c_{N \alpha}(k ; z)=\left\{\begin{array}{llll}
\geqslant 0 & \text { при } & \mu_{N \alpha} \in\left(0, \mu_{N \alpha}^{c}(k)\right] \quad \text { для всякого } z \in(-\infty, m(k)], \\
<0 & \text { при } & \mu_{N \alpha}>\mu_{N \alpha}^{c}(k), \quad z=m(k), \\
1 & \text { при } & z \rightarrow-\infty,
\end{array}\right. \\
1-\mu_{N \alpha} s_{N \alpha}(k ; z)=\left\{\begin{array}{llll}
\geqslant 0 & \text { при } & \mu_{N \alpha} \in\left(0, \mu_{N \alpha}^{s}(k)\right] \quad \text { для всякого } z \in(-\infty, m(k)], \\
<0 & \text { при } & \mu_{N \alpha}>\mu_{N \alpha}^{s}(k), \quad z=m(k), \\
1 & \text { при } & z \rightarrow-\infty .
\end{array}\right.
\end{aligned}
$$

Заметим, что функции $d(k ; \cdot), c_{N \alpha}(k ; \cdot), s_{N \alpha}(k ; \cdot)$, определенные по формулам (2), являются положительными, монотонно возрастающими на $(-\infty, m(k))$. Поэтому из последних соотношений имеем

$$
\begin{array}{r}
1-\mu_{l \alpha} d(k ; \cdot)=\left\{\begin{array}{lll}
\text { не имеет нулей } & \text { при } & \mu_{l \alpha} \in\left(0, \mu^{0}(k)\right], \\
\text { имеет единственный нуль } & \text { при } & \mu_{l \alpha}>\mu^{0}(k),
\end{array}\right. \\
1-\mu_{N \alpha} c_{N \alpha}(k ; \cdot)=\left\{\begin{array}{lll}
\text { не имеет нулей } & \text { при } & \mu_{N \alpha} \in\left(0, \mu_{N \alpha}^{c}(k)\right], \\
\text { имеет единственный нуль } & \text { при } & \mu_{N \alpha}>\mu_{N \alpha}^{c}(k),
\end{array}\right. \\
1-\mu_{N \alpha} s_{N \alpha}(k ; \cdot)=\left\{\begin{array}{lll}
\text { не имеет нулей } & \text { при } & \mu_{N \alpha} \in\left(0, \mu_{N \alpha}^{s}(k)\right], \\
\text { имеет единственный нуль } & \text { при } & \mu_{N \alpha}>\mu_{N \alpha}^{s}(k) .
\end{array}\right.
\end{array}
$$

Отсюда и согласно леммам 4, 2 получаем утверждение теоремы 1.

ДоКАЗАТЕЛЬСТво тЕОремЫ 2. Пусть выполняется предположение 1 и $k \in \Pi$, $k_{\alpha}= \pm \pi / 2 n$. В этом случае функция $\widetilde{\mathcal{E}}_{k}(\cdot)$ не зависит от $p_{\alpha}$. Поэтому имеет место

$$
\lim _{z \rightarrow m(k)} d(k ; z)=+\infty
$$

Отсюда для всякого $\mu_{l \alpha}>0$ получим

$$
\lim _{z \rightarrow m(k)}\left(1-\mu_{l \alpha} d(k ; z)\right)=-\infty, \quad \lim _{z \rightarrow-\infty}\left(1-\mu_{l \alpha} d(k ; z)\right)=1 .
$$

Из непрерывности и монотонности функции $d(k ; \cdot)$ следует, что функция $1-\mu_{l \alpha} d(k ; \cdot)$ имеет единственный нуль на $(-\infty, m(k))$. Следовательно, функция $\Delta(k ; \cdot)$ имеет $6 N$ нулей для всякого $\mu \in \mathbb{R}_{+}^{3 N}, \mu=\left(\mu^{(1)}, \ldots, \mu^{(N)}\right), \mu^{(l)}=\left(\mu_{l 1}, \mu_{l 2}, \mu_{l 3}\right)$.

Согласно леммам 4, 2 получаем утверждение теоремы 2. 


\section{4. ВИРТУАЛЬНЫЙ УРОВЕНЬ ОПЕРАТОРА $H(0)$}

В этом разделе докажем теорему 3. По определению виртуального уровня оператора $h(\mathbf{0})$ будем изучать уравнение

$$
G \varphi=\varphi
$$

Отметим, что функция $\Delta(\mathbf{0} ; \cdot)$ определена при $z=0$. Аналогично лемме 4 доказывается

Лемма 5. Число $\lambda=1$ является собственным значением оператора $G$ тогда и только тогда, когда

$$
\Delta(\mathbf{0} ; 0)=0 .
$$

При этом если $1-\mu_{l \alpha} d(\mathbf{0} ; 0)=0\left(1-\mu_{N \alpha} c_{N \alpha}(\mathbf{0} ; 0)=0\right)$, то функции $\varphi_{l \alpha}(p)=$ $\cos l p_{\alpha}, \psi_{l \alpha}(p)=\sin l p_{\alpha}, l \in\{1, \ldots, N\}\left(\varphi_{N \alpha}(p)=\cos N p_{\alpha}\right)$ являются собственными функииями оператора $G$, соответствующими собственному значению 1.

ДоКАЗАТЕЛЬСТВо ТЕОРЕМЫ 3. Если $n / 2 N$ - натуральное (дробное) число, $\mu_{l i} \in\left[0, \mu^{0}(\mathbf{0})\right)\left(\mu_{l i} \in\left[0, \mu^{0}(\mathbf{0})\right), \mu_{N i} \in\left[0, \mu_{N}^{0}(\mathbf{0})\right)\right)$ для каждого $l \in\{1, \ldots, N\}(l \in$ $\{1, \ldots, N-1\})$ и $i \in\{1,2,3\}$, то $\Delta(\mathbf{0} ; 0)>0$. В этом случае из леммы 5 следует, что число $\lambda=1$ не является собственным значением оператора $G$.

Если $n / 2 N$ - натуральное число и $\mu_{l \alpha}=1 / d(\mathbf{0} ; 0)$, то из леммы 5 следует, что число $\lambda=1$ является собственным значением оператора $G$, а $\varphi_{l \alpha}(p)=\cos l p_{\alpha}$ и $\psi_{l \alpha}(p)=\sin l p_{\alpha}$ являются соответствующими собственными функциями оператора $G$. Поэтому

$$
h(\mathbf{0}) f(p)=0, \quad h(\mathbf{0}) g(p)=0,
$$

где $f(p)=\cos l p_{\alpha} / \mathcal{E}_{\mathbf{0}}(p), g(p)=\sin l p_{\alpha} / \mathcal{E}_{\mathbf{0}}(p)$. Легко проверить, что $f \in L_{1}\left(\mathbb{T}^{3}\right) \backslash$ $L_{2}\left(\mathbb{T}^{3}\right)$ и $g \in L_{2}\left(\mathbb{T}^{3}\right)$, т. е. $z=0$ является одновременно и виртуальным уровнем, и собственным значением оператора $h(\mathbf{0})$.

Если $n / 2 N-$ дробное число и $\mu_{N \alpha}=\mu_{N}^{0}(\mathbf{0})$, то

$$
1-\mu_{N \alpha} c_{N \alpha}(\mathbf{0} ; 0)=0, \quad 1-\mu_{N \alpha} s_{N \alpha}(\mathbf{0} ; 0)>0 .
$$

Следовательно, число $\lambda=1$ является собственным значением оператора $G$, соответствующим собственной функции $\varphi_{N \alpha}(p)=\cos N p_{\alpha}$ и

$$
F(p)=\frac{\cos N p_{\alpha}}{\mathcal{E}_{\mathbf{0}}(p)} \in L_{1}\left(\mathbb{T}^{3}\right) \backslash L_{2}\left(\mathbb{T}^{3}\right),
$$

т. е. $z=0$ является виртуальным уровнем оператора $h(\mathbf{0})$. Теорема доказана.

\section{Список литературы}

[1] Л. Д. Фаддеев, Тр. МИАН, 69 (1963), 3-122.

[2] D. C. Mattis, Rev. Modern Phys., 58:2 (1986), 361-379.

[3] S. Albeverio, S. N. Lakaev, K. A. Makarov, Z. I. Muminov, Commun. Math. Phys., 262:1 (2006), 91-115.

[4] Д. Р. Яфаев, Матем. сб., 94(136):4(8) (1974), 567-593. 
[5] A. V. Sobolev, Commun. Math. Phys., 156:1 (1993), 101-126.

[6] Д. Р. Яфаев, ТМФ, 25:2 (1975), 185-195.

[7] С. А. Вугальтер, Г. М. Жислин, Тр. ММО, 49 (1986), 95-112.

[8] Г. М. Жислин, ТМФ, 68:2 (1986), 265-275.

[9] С. Н. Лакаев, ТМФ, 89:1 (1991), 94-104.

[10] С. Н. Лакаев, М.Э. Муминов, ТМФ, 135:3 (2003), 478-503.

[11] Е. Л. Лакштанов, Р. А. Минлос, Функи. анализ и его прилож., 39:1 (2005), 39-55.

[12] P. A. Faria da Veiga, L. Ioriatti, M. O'Carroll, Phys. Rev. E, 66:1 (2002), 016130, 9 pp.

[13] М.Э. Муминов, ТМФ, 153:3 (2007), 381-387.

[14] М.Э. Муминов, А. М. Хуррамов, ТМФ, 177:3 (2013), 482-496.

[15] М.Э. Муминов, 159:2 (2009), 299-317.

[16] М. Рид, Б. Саймон, Методы современной математической физики, т. 4: Анализ операторов, Мир, М., 1982. 